\title{
Tratamento de superfície para a cimentação adesiva de cerâmicas à base de zircônia: revisão de literatura
}

\section{Surface treatment for the adhesive cementation of zirconia-based ceramics: a literature review}

Paula Fernanda Gomes de Oliveira, ${ }^{1}$ Tiago Braga Rabello ${ }^{1}$

${ }^{1}$ Departamento de Clínica Odontológica, Faculdade de Odontologia, Universidade Federal do Rio de Janeiro, Rio de Janeiro, RJ, Brasil

- Os autores declaram que não há conflito de interesse.

\section{Resumo}

Objetivo: analisar, por meio de uma revisão da literatura, os diferentes métodos de tratamento de superfície de peças protéticas à base de zircônia e os seus efeitos sobre a cimentação adesiva.

Material e Métodos: a seleção dos artigos foi realizada por meio de uma busca na base de dados PubMed/MEDLINE. A amostra final foi composta por 31 estudos publicados entre 2011-2016. Resultados: as cerâmicas à base de zircônia $\left(\mathrm{ZrO}_{2}\right)$ têm propriedades importantes, como durabilidade química, alta tenacidade à fratura, módulo de elasticidade e dureza. Apesar da dificuldade de ligação da superfície da $\mathrm{ZrO}_{2}$ aos cimentos resinosos, estes são uma boa opção para unir as restaurações indiretas aos tecidos dentários. Entretanto, o condicionamento com ácido hidrofluorídrico e a silanização não melhoram a força de união entre o cimento resinoso convencional e cerâmica de alto conteúdo cristalino. Por esta razão, é necessário um modo de tratamento de superfície diferente para proporcionar uma força de ligação adequada. Conclusão: os estudos disponíveis sugerem que o jateamento com partículas de óxido de alumínio e a silicatização proporcionam rugosidade superficial e uma adequada resistência adesiva, no entanto, induzem à transformação de fase tetragonal em monoclínica. Por outro lado, a infiltração seletiva por vidro demonstra não gerar danos à superfície da ZrO2, porém, há a necessidade de mais pesquisas sobre esse recente método.

Palavras-chave: Zircônia; Tratamento de superfície; Cimento resinoso.

Abstract

Objective: tanalyze, through a literature review, the current surface treatments and their effect on the adhesive cementation of zirconia prosthetic parts. Material and Methods: a search was conducted in the PubMed database. The final sample consisted of 31 studies published between 2011 and 2016 . Results: zirconia-based ceramics $\left(\mathrm{ZrO}_{2}\right)$ have important properties, such as chemical durability, high fracture toughness, elastic modulus, and hardness. Despite the difficulty in bonding the surface of $\mathrm{ZrO}_{2}$, resin cements are a good option to bond indirect restorative materials to dental tissues. Hydrofluoric etching and silanation do not improve the bond strength between conventional resin cement and high crystalline content ceramics. For this reason, a different mode of treatment is necessary to provide adequate bond strength. Conclusion: the results of most available studies suggest that blasting and silicatization provide surface roughness and good adhesive strength; however, they induce the transformation of tetragonal/ monoclinic phases. The selective infiltration by glass demonstrates no damage to the surface of the $\mathrm{ZrO}_{2}$; more research is needed on this new method.

Keywords: Zirconium; Surface treatment; Resin cement.

\section{Introdução}

a m 1956, visando melhorar a resistência das restaurações cerâmicas, Brecker introduziu as restaurações metalocerâmicas na Odontologia. A infraestrutura metálica não permitia que a cerâmica sofresse flexão, aumentando acentuadamente a resistência nas áreas onde houvesse incidência direta de força mastigatória. Por outro lado, a presença de infraestrutura metálica e a necessidade do uso de opacificadores eram limitantes à transmissão de luz, o que, muitas vezes, comprometia o resultado estético. ${ }^{1}$

$\mathrm{Na}$ tentativa de solucionar esse problema, foram desenvolvidas novas tecnologias buscando materiais cerâmicos com resistência suficiente para serem empregados em áreas de elevada força mastigatória sem a necessidade de haver uma subestrutura metálica. As pesquisas na ciência de materiais cerâmicos para aplicações dentárias levaram ao desenvolvimento de uma classe de materiais de alta resistência: as cerâmicas à base de zircônia. ${ }^{2}$

A zircônia é um material dinâmico e apresenta três formas cristalinas (cúbica, tetragonal e monoclínica) de acordo com variações de temperatura. Diante deste fato, há a necessidade da adição de 3-6\% em peso de um agente estabilizante. Na Odontologia, utiliza-se o ítrio, produzindo uma zircônia tetragonal parcialmente estabilizada (Y-TZP) em temperatura ambiente. ${ }^{3} \mathrm{~A}$ resistência à flexão e a tenacidade à fratura são consideravelmente maiores do que as de outras cerâmicas dentárias e a cerâmica à base de zircônia apresenta um mecanismo distinto de resistência denominado transformação induzida pelo estresse, o que significa que o material sofre alterações microestruturais quando submetido ao estresse. A cerâmica à base de zircônia pode resistir ativamente à propagação de rachaduras pela transformação de uma fase tetragonal em uma fase monoclínica na ponta de uma fenda, que é acompanhada por um aumento de volume de $3 \%$ a $4 \%{ }^{4}$

Apesar de apresentar desempenho mecânico superior, uma das principais limitações das cerâmicas à base de zircônia está relacionada à sua cimentação as estruturas dentárias. ${ }^{5} \mathrm{~A}$ princípio, a cimentação convencional foi recomendada. No entanto, a aplicação da cimentação adesiva proporciona elevada retenção, melhora a adaptação marginal, evita a microinfiltração, fornece resistência à fratura do dente e da restauração, e melhora a longevidade da cerâmica. Além disso, a cimentação adesiva oferece a vantagem de selar pequenas falhas na superfície interna criadas por corrosão ou jateamento de partículas, reforçando significativamente o material cerâmico. ${ }^{6,7}$

Embora o uso da zircônia esteja, atualmente, bem estabelecido na Odontologia, não há um consenso sobre o protocolo ideal para a cimentação adesiva destas peças protéticas. ${ }^{8}$ Durante os últimos quinze anos, os estudos têm se concentrado na busca por diferentes estratégias para se obter uma adequada cimentação adesiva 
para as cerâmicas à base de zircônia. Entretanto, a ausência de conteúdo de sílica na composição deste tipo de cerâmica dificulta o alcance deste objetivo. ${ }^{9}$

Nesse contexto, o propósito desse trabalho foi analisar, por meio de uma revisão da literatura, os diferentes métodos de tratamento de superfície de peças protéticas à base de zircônia e os seus efeitos sobre a cimentação adesiva.

\section{Material e Métodos}

Foi realizado um levantamento bibliográfico a partir de uma busca eletrônica na base de dados Pubmed/MEDLINE. Os termos utilizados para a busca, segundo o Medical Subject Headings (MeSH), foram: zirconium, surface treatment, surface treatments, shear strength, shear strengths, tensile strength, tensile strengths, resin cement, resin cements. A pesquisa restringiu-se a estudos realizados no período de 2011 a 2016, disponíveis online, nos idiomas português, inglês e espanhol. Foram obtidos no total 38 artigos; dentre eles, foram excluídos sete artigos, pois não apresentavam relação com o tema deste trabalho.

\section{Revisão da Literatura/Discussão}

Nas cerâmicas vítreas, o condicionamento com ácido hidrofluorídrico é utilizado para modificar a superfície interna da peça protética. Este ácido remove seletivamente a matriz vítrea, tornando a superfície porosa. Após o condicionamento ácido, um agente silano é aplicado sobre a superfície da cerâmica. O silano é uma molécula bifuncional capaz de formar ligações siloxanas com os grupamentos hidroxila da sílica na superfície cerâmica e copolimerizar com a matriz resinosa dos cimentos adesivos. Entretanto, esta reação química não é aplicável às cerâmicas à base de zircônia devido à ausência de sílica. ${ }^{8,10-13}$

O tratamento de superfície ideal para as cerâmicas à base de zircônia é motivo de apreensão até os dias atuais. Não há unanimidade quanto ao melhor condicionamento superficial e inúmeros tratamentos de superfície têm sido descritos na literatura para cerâmicas à base de zircônia. ${ }^{14}$

$\mathrm{O}$ jateamento com partículas de óxido de alumínio $\left(\mathrm{Al}_{2} \mathrm{O}_{3}\right)$ é um dos métodos preferencialmente descrito. ${ }^{2}$ Este método proporciona o aumento da energia livre de superfície melhorando a molhabilidade. Além disso, aumenta a rugosidade da superfície possibilitando melhor embricamento mecânico do cimento resinoso, ${ }^{8,9,15}$ essa retenção mecânica é essencial não apenas para estabelecer elevadas forças de união, mas também para evitar falhas adesivas. ${ }^{16}$ No entanto, o jateamento induz a transformação da fase tetragonal para a fase monoclínica, desencadeando a propagação de microfissuras na cerâmica durante a função e isso diminui o desempenho a longo prazo da restauração em cerca de 20 a $30 \% .^{2,6,8-10,15,17,18}$

Shin et al. ${ }^{11}$ avaliaram o efeito de diferentes tratamentos de superfície sobre a força de união de dois diferentes cimentos resinosos contendo monômeros fosfatados à uma cerâmica à base de zircônia. Os autores concluíram que o jateamento com partículas de óxido de alumínio seguido da aplicação de um primer para zircônia alcançou o melhor resultado de união. Da mesma forma, Zandparsa et al. ${ }^{19}$ observaram que o jateamento seguido da aplicação de um primer (Z-PRIME Plus - Bisco, Inc., Schaumburg, IL) produziu uma maior força de união, capaz de resistir à degradação ao longo do tempo. Além disso, os autores salientam que a zircônia tratada com o jateamento apresenta uma ligação comparável àquela obtida entre o cimento resinoso e o esmalte.

Komine et al..$^{20}$ relataram em seu estudo que o jateamento a uma pressão de $0,1 \mathrm{MPa}$ ou superior aumenta a força de união entre a cerâmica e o cimento resinoso. Entretanto, o meio mais eficaz de se realizar o jateamento é ainda incerto. As variáveis "tamanho de partículas" e "pressão do jato" e o efeito desses sobre a adesão e sobre as propriedades mecânicas da zircônia devem ser investigados. ${ }^{17}$

Outro tratamento de superfície para as cerâmicas à base de zircônia descrito na literatura é a silicatização. Neste método, as partículas de óxido de alumínio modificados por sílica são jateadas sobre a superfície cerâmica. O impacto das partículas gera um triboplasma e a consequente incorporação de sílica à superfície. ${ }^{11}$ Este processo não apenas cria irregularidades superficiais, favorecendo a retenção micromecânica, como também recobre a superfície cerâmica por sílica, o que permite o processo de silanização. ${ }^{8,11}$

Mohmoodi et al. ${ }^{21}$ avaliaram o efeito de três diferentes métodos de tratamento de superfície sobre a força de união de dois cimentos resinosos à base de 10-MDP à uma cerâmica à base de zircônia Os autores concluíram que a silicatização seguida de silanização da superfície da cerâmica melhorou significativamente a força de união para os dois cimentos resinosos.

Contudo, tem sido demostrado que incorporação de sílica à superfície da cerâmica é excessivamente baixa para uma silanização eficaz ${ }^{22}$ e que há uma significativa redução da força de união, após o envelhecimento artificial, uma vez que a ligação siloxana é sensível à degradação hidrolítica, afetando assim a estabilidade dessa ligação. ${ }^{23-26}$

Outro método que tem sido utilizado para o tratamento da superfície das cerâmicas à base de zircônia é a aplicação de primes e cimentos resinosos que contenham em sua composição o monômero fosfatado 10-MDP, capaz de estabelecer uma interação química com os grupos hidroxila dos óxidos metálicos da zircônia sem qualquer pré-tratamento e desenvolver uma união estável. ${ }^{9,11,17,18,26}$

Subasi e Inan ${ }^{12}$ observaram em seu estudo que a seleção do cimento é considerada mais importante do que o tipo de tratamento de superfície e que os cimentos contendo monômeros fosfatados foram adequados para a cimentação da zircônia. Apesar disso, estudos demonstram que o uso de primers e cimentos contendo o monômero 10-MDP associado ao jateamento de partículas ou à silicatização tem produzido uma força de união mais elevada do que o seu uso sem tratamento de superfície, pois essas modificações superficiais além de gerarem mais grupos hidroxila livres para a interação com os monômeros 10-MDP, proporcionam também uma retenção micromecânica. ${ }^{11,12,27,28}$

Da Silva et al. ${ }^{18}$ salientaram que, apesar de estudos recentes preconizarem a utilização de primers metálicos contendo 10MDP com o objetivo de promover a ligação entre as cerâmicas à base de zircônia e os cimentos resinosos, é importante a criação de microrretenções e de alta energia de superfície para melhorar e estabilizar essa união. O resultado do estudo de Saker et al ${ }^{29}$ 
corrobora com esta afirmação. Estes autores verificaram que o jateamento da superfície previamente à cimentação adesiva com cimentos resinosos à base de 10-MPD aumenta a força de união entre a cerâmica à base de zircônia e o cimento.

Outro método descrito na literatura para tornar rugosa a superfície interna da peça protética à base de zircônia é a irradiação por laser. Diferentes tipos de lasers têm sido utilizados na Odontologia. ${ }^{14}$

O laser Nd:YAG, indicado para o tratamento da hipersensibilidade dentinária, remoção de cárie e clareamento dentário, é uma opção para o tratamento de superfície de cerâmicas à base de zircônia. Liu et al. ${ }^{10}$ realizaram um estudo onde investigaram o efeito da irradiação pelo laser Nd:YAG com diferentes potências de saída e tempos de irradiação. Os autores concluíram que a irradiação é capaz alterar as características morfológicas da superfície cerâmica. O laser Er:YAG tem aplicações semelhantes ao Nd:YAG, incluindo remoção de tecido cariado, preparo de cavidades e modificação da superfície cerâmica. Este apresenta a capacidade de remover partículas por um processo chamado "ablação", que inclui microexplosões. ${ }^{12,14}$ Outro laser utilizado é o $\mathrm{CO}_{2}$, apropriado para o tratamento de superfície da cerâmica devido ao seu comprimento de onda, que é totalmente absorvido pelo material. ${ }^{14}$

Akin et al. ${ }^{15}$ avaliaram o efeito de diferentes tratamentos de superfície. Os autores concluíram que os tratamentos de superfície com os lasers Er:YAG e Nd:YAG apresentaram maior força de união entre a zircônia e o cimento se comparados ao jateamento com óxido de alumínio e o tratamento com o laser $\mathrm{CO}_{2}$. No entanto, Erden et al. ${ }^{6}$ observaram, em seu estudo, que o tratamento com laser não melhora a força de união entre o cimento resinoso e a cerâmica à base de zircônia. Estudos mostram que há pouca informação sobre a avaliação do efeito de diferentes potências de saída e tempos de irradiação desses lasers e que uma elevada irradiação pode induzir a transformação da fase tetragonal para a monoclínica. ${ }^{10,30}$

A infiltração seletiva por vidro é um novo método para o tratamento da zircônia e tem sido desenvolvido para transformar a superfície densa deste material em uma superfície microporosa capaz de interagir com o cimento resinoso. ${ }^{31} \mathrm{O}$ método emprega um processo de maturação induzida por calor que permite a infiltração de vidro fundido entre os grãos de zircônia. Logo após, a superfície é condicionada com o ácido hidrofluorídrico, criando uma topografia superficial rugosa e permitindo o embricamento nanomecânico do cimento resinoso. Estudos têm evidenciado um aumento significativo da rugosidade da superfície sem sinais de degradação da cerâmica, tornando este tratamento promissor para o condicionamento da zircônia. ${ }^{9}$

\section{Conclusão}

Inúmeras técnicas para o tratamento da superfície estão descritas na literatura com a pretensão de alcançar uma resistência adesiva ideal para a cimentação adesiva da zircônia. Um tratamento de superfície não deve gerar um efeito prejudicial às propriedades da zircônia. Mesmo proporcionando rugosidade superficial e uma boa resistência adesiva, o jateamento com partículas de óxido de alumínio e a silicatização são capazes de induzir a transformação da fase tetragonal, o que pode estabelecer fraturas na zircônia em longo prazo. A infiltração seletiva por vidro parece ser um método bastante promissor, pois promete ser capaz de gerar uma ligação química e embricamento nanomecânico sem que haja danos na superfície cerâmica. No entanto, há necessidade de mais pesquisas sobre esse novo tratamento.

\section{Referências}

1. Vernekar NV, Jagadish PK, Diwakar S, Nadgir R, Krishnarao MR. Alternate metal framework designs for the metal ceramic prosthesis to enhance the esthetics. J Adv Prosthodont. 2011;3(3):113-8.

2. Zhang Y, Sun T, Liu R, Feng X, Chen A, Shao L. An in vitro evaluation of the zirconia surface treatment by mesoporous zirconia coating on its bonding to resin cement. Biomed Mater Eng. 2014;24(6):2109-16.

3. Akin H, Tugut F, Akin GE, Guney U, Mutaf B. Effect of Er:YAG laser application on the shear bond strength and microleakage between resin cements and Y-TZP ceramics. Lasers Med Sci. 2012;27(2):333-8.

4. Valentino TA, Borges GA, Borges LH, Platt JA, Correr-Sobrinho L. Influence of glazed zirconia on dual-cure luting agent bond strength. Oper Dent. 2012;37(2):181-7.

5. Martins AR, Gotti VB, Shimano MM, Borges GA, Goncalves Lde S. Improving adhesion between luting cement and zirconia-based ceramic with an alternative surface treatment. Braz Oral Res. 2015;29:54.

6. Erdem A, Akar GC, Kose T. Effects of different surface treatments on bond strength between resin cements and zirconia ceramics. Oper Dent. 2014;39(3):E118-27.

7. Casucci A, Goracci C, Chieffi N, Monticelli F, Giovannetti A, Juloski J, et al. Microtensile bond strength evaluation of self-adhesive resin cement to zirconia ceramic after different pre-treatments. Am J Dent. 2012;25(5):269-75.

8. Menani LR, Farhat IA, Tiossi R, Ribeiro RF, Guastaldi AC. Effect of surface treatment on the bond strength between yttria partially stabilized zirconia ceramics and resin cement. J Prosthet Dent. 2014;112(2):357-64.

9. Samimi P, Hasankhani A, Matinlinna JP, Mirmohammadi H. Effect of Adhesive Resin Type for Bonding to Zirconia Using Two Surface Pretreatments. J Adhes Dent. 2015;17(4):353-9.

10. Liu L, Liu S, Song X, Zhu Q, Zhang W. Effect of Nd: YAG laser irradiation on surface properties and bond strength of zirconia ceramics. Lasers Med Sci.
2015;30(2):627-34.

11. Shin YJ, Shin Y, Yi YA, Kim J, Lee IB, Cho BH, et al. Evaluation of the shear bond strength of resin cement to Y-TZP ceramic after different surface treatments. Scanning. 2014;36(5):479-86.

12. Subasi MG, Inan O. Influence of surface treatments and resin cement selection on bonding to zirconia. Lasers Med Sci. 2014;29(1):19-27.

13. de Castro HL, Corazza PH, Paes-Junior Tde A, Della Bona A. Influence of Y-TZP ceramic treatment and different resin cements on bond strength to dentin. Dent Mater. 2012;28(11):1191-7.

14. El-Korashy DI, El-Refai DA. Mechanical properties and bonding potential of partially stabilized zirconia treated with different chemomechanical treatments. J Adhes Dent. 2014;16(4):365-76.

15. Akin H, Ozkurt Z, Kirmali O, Kazazoglu E, Ozdemir AK. Shear bond strength of resin cement to zirconia ceramic after aluminum oxide sandblasting and various laser treatments. Photomed Laser Surg. 2011;29(12):797-802.

16. Aboushelib MN. Fusion sputtering for bonding to zirconia-based materials. J Adhes Dent. 2012;14(4):323-8.

17. Re D, Augusti D, Augusti G, Giovannetti A. Early bond strength to low-pressure sandblasted zirconia: evaluation of a self-adhesive cement. Eur J Esthet Dent. 2012;7(2):164-75.

18. da Silva EM, Miragaya L, Sabrosa CE, Maia LC. Stability of the bond between two resin cements and an yttria-stabilized zirconia ceramic after six months of aging in water. J Prosthet Dent. 2014;112(3):568-75.

19. Zandparsa R, Talua NA, Finkelman MD, Schaus SE. An in vitro comparison of shear bond strength of zirconia to enamel using different surface treatments. J Prosthodont. 2014;23(2):117-23.

20. Komine F, Fushiki R, Koizuka M, Taguchi K, Kamio S, Matsumura H. Effect of surface treatment on bond strength between an indirect composite material and a zirconia framework. J Oral Sci. 2012;54(1):39-46. 
21. Mahmoodi N, Hooshmand T, Heidari S, Khoshro K. Effect of sandblasting, silica coating, and laser treatment on the microtensile bond strength of a dental zirconia ceramic to resin cements. Lasers Med Sci. 2016;31(2):205-11.

22. Perdigao J, Fernandes SD, Pinto AM, Oliveira FA. Effect of artificial aging and surface treatment on bond strengths to dental zirconia. Oper Dent. 2013;38(2):168-76

23. Kim JH, Chae S, Lee Y, Han GJ, Cho BH. Comparison of shear test methods for evaluating the bond strength of resin cement to zirconia ceramic. Acta Odontol Scand. 2014;72(8):745-52.

24. Cheung GC, Botelho MG, Matinlinna JP. Effect of surface treatments of zirconia ceramics on the bond strength to resin cement. J Adhes Dent. 2014;16(1):49-56. 25. Piascik JR, Swift EJ, Braswell K, Stoner BR. Surface fluorination of zirconia: adhesive bond strength comparison to commercial primers. Dent Mater. 2012;28(6):604-8.

26. Sciasci P, Abi-Rached FO, Adabo GL, Baldissara P, Fonseca RG. Effect of sur- face treatments on the shear bond strength of luting cements to Y-TZP ceramic. J Prosthet Dent. 2015;113(3):212-9.

27. Baldissara P, Querze M, Monaco C, Scotti R, Fonseca RG. Efficacy of surface treatments on the bond strength of resin cements to two brands of zirconia ceramic. J Adhes Dent. 2013;15(3):259-67.

28. Qeblawi DM, Campillo-Funollet M, Munoz CA. In vitro shear bond strength of two self-adhesive resin cements to zirconia. J Prosthet Dent. 2015;113(2):122-7. 29. Saker S, Ibrahim F, Ozcan M. Effect of different surface treatments on adhesion of In-Ceram Zirconia to enamel and dentin substrates. J Adhes Dent. 2013;15(4):369-76.

30. Akpinar YZ, Kepceoglu A, Yavuz T, Aslan MA, Demirtag Z, Kilic HS, et al. Effect of femtosecond laser beam angle on bond strength of zirconia-resin cement. Lasers Med Sci. 2015;30(8):2123-8.

31. Cheung GJ, Botelho MG. Zirconia Surface Treatments for Resin Bonding. J Adhes Dent. 2015;17(6):551-8.

\section{Mini Currículo e Contribuição dos Autores}

1. Paula Fernanda Gomes de Oliveira - cirurgiã-dentista e especialista. Contribuição: aquisição e interpretação dos dados, preparação e redação do manuscrito. 2. Tiago Braga Rabello - cirurgião-dentista e PhD. Contribuição: participação científica e intelectual efetiva para o estudo, concepção e delineamento, revisão crítica e aprovação final.

Recebido em: 12/01/2017 / Aprovado em: 10/02/2017

Autor Correspondente

Tiago Braga Rabello

E-mail: tiagobragarabello@gmail.com 